\title{
CROSS-SECTIONS OF SOLUTION FUNNELS
}

\author{
BY CHARLES C. PUGH ${ }^{1}$ \\ Communicated by L. Markus, March 30, 1964
}

1. Consider $R^{n+1}$ as $(t, y)$-space where $t$ is real and $y=\left(y^{1}, \cdots, y^{n}\right)$ $\in R^{n}$. Let $\mathcal{F}^{n}$ denote the set of all continuous maps $f: R^{n+1} \rightarrow R^{n}$ having compact support. For any $p=\left(t_{0}, y_{0}\right) \in R^{n+1}$ and $f \in \mathcal{F}^{n}$, an $f$-solution through $p$ is any $C^{1}$ map $y: R \rightarrow R^{n}$ such that $y(t)$ is a solution of the initial value problem

$$
\frac{d y(t)}{d t}=f(t, y(t)), \quad y\left(t_{0}\right)=y_{0} .
$$

The f-funnel through $p, F(p)$, is the union of all the curves $(t, y(t))$ in $R^{n+1}$ such that $y(t)$ is an $f$-solution. E. Kamke [3] introduced the term integraltrichter in 1932. (When $f$ is Lipschitz continuous, then of course $F(p)$ is just the unique $f$-solution curve through $p$, but if $f$ is only $C^{0}$ then $F(p)$ may consist of many $f$-solution curves.) For any real number $s$, the cross-section of $F(p)$ at time $s$ is the set $K_{s}(p)$ $=\left\{y \in R^{n}:(s, y) \in F(p)\right\}$.

Definition. A subset $A$ of $R^{m}$ is a funnel-section if for some $n \geqq m$ there exist $f \in \mathscr{F}^{n}$ and $p \in R^{n+1}$ such that $i(A)=K_{s}(p)$ for some real $s$, where $i: R^{m} \rightarrow R^{n}$ is the usual injection of $R^{m}$ onto the span of the first $m$ coordinate axes of $R^{n}$.

2. A theorem of $\mathrm{H}$. Kneser [5] asserts that any funnel-section is a continuum (i.e., a compact, connected set). There naturally arises, then, the question: what are necessary and sufficient conditions that a continuum in $R^{m}$ be a funnel-section? We prove the six theorems below as partial answers to this question.

Theorem 1. There exists a continuum $P$ which is not a funnelsection.

THEOREM 2. There exists a funnel-section $S$ which is not arcwise connected.

$P$ is a bounded outward spiral in $\mathrm{C}=R^{2}$ together with its limit circle:

1 The author holds a fellowship from the United States Steel Foundation at The Johns Hopkins University. This research was also supported in part by the Air Force Office of Scientific Research. I am glad to thank Dr. Philip Hartman for his patience and helpful advice. 
$P=\left\{z \in C: z=(1-1 / \theta) e^{i \theta}\right.$ for $\left.2 \pi \leqq \theta<\infty\right\} \cup\{z \in C:|z|=1\}$.

$S$ is a continuum of the same form as

$$
\left\{(x, y) \in[-1,1]^{2}: x \neq 0 \text { implies } y=\sin (1 / x)\right\} .
$$

Definition. A $C^{1}$ polyhedron is the image of a finite abstract polyhedron that has been imbedded in Euclidean space by a map which is bi- $C^{1}$ on each simplex.

Theorem 3. Any $C^{1}$ polyhedron is a funnel-section.

Theorem 3 implies that all $C^{1}$ manifolds, algebraic varieties, and rectilinear polyhedra are funnel-sections. In particular, funnel-sections may fail to be simply connected as was previously observed by M. Nagumo and M. Fukuhara in [8].

3. Definition. Let $f$ be in $\mathscr{F}^{n}$ and let $Z$ be any subset of $R^{n+1}$. The funnel of $f$-solutions through $Z$ is defined as

$$
F(Z)=\bigcup_{p \in Z} F(p)
$$

and the cross-section of $F(Z)$ at time $s$ is defined to be

$$
K_{s}(Z)=\left\{y \in R^{n}:(s, y) \in F(Z)\right\} .
$$

This notion of the funnel of $f$-solutions through a set (instead of just through a point) seems first to have been defined by M. Fukuhara in [2]. As he points out (Theorem 2 in [2]), it is easy to generalize Kneser's Theorem by replacing $p$ with a continuum $Z$. In our terminology, a funnel-section is the cross-section of a funnel through a point.

Definition. Let $f$ be in $\Im^{n}$ and let $A$ be a subset of $R^{n}$. Let $a$ and $b$ be real numbers. We define $F(a \times A)$ to be $(a, b)$-stable if $A=K_{a}\left(b \times K_{b}(a \times A)\right)$. (This means that if $y_{0} \in A$ and if $y(t)$ is any $f$-solution through $\left(a, y_{0}\right)$ and if $\tilde{y}(t)$ is any $f$-solution through $(b, y(b))$, then $\tilde{y}(a) \in A$.)

Theorem 4. Let $f$ be in $\mathfrak{F}^{n}$ and let $A$ be compact. Suppose that $F(a \times A)$ is $(a, b)$-stable for some $a, b \in R$. Then $R^{n}-A$ is diffeomorphic to $R^{n}-K_{b}(a \times A)$ by a diffeomorphism which is the identity on some neighborhood of infinity (i.e. the complement of some compact set).

The full converse of Theorem 4 is false (which we can show by an example). However, we can prove

Theorem 5. Suppose $A$ is a continuum in $R^{n}$ such that $R^{n}-A$ is diffeomorphic to $R^{n}-0$ by a diffeomorphism which is the identity on a neighborhood of infinity. Then $A$ is a stable funnel-section, i.e., there 
exists $f \in \mathcal{F}^{n}$ and $y_{0} \in R^{n}$ such that, for $p=\left(0, y_{0}\right), F(p)$ is $(0,1)$-stable and $K_{1}(p)=A$.

Theorems 4 and 5 completely characterize stable funnel-sections.

4. A stronger version of Theorem 5 would be

Theorem $5^{\prime}$. If $A$ is a continuum in $R^{n}$ such that $R^{n}-A$ is diffeomorphic to $R^{n}-0$, then $A$ is a stable funnel-section.

To deduce Theorem $5^{\prime}$ directly from Theorem 5 , we should need the following proposition from topology.

Proposition 1. If $A$ is a continuum in $R^{n}$ such that $R^{n}-A$ is diffeomorphic to $R^{n}-0$, then there is a diffeomorphism $f: R^{n}-A \rightarrow R^{n}-0$ which is the identity in a neighborhood of infinity.

This proposition can be proved using the $C^{\infty}$ Schönflies Conjecture which asserts that if $f: S^{n-1} \rightarrow S^{n}$ is a $C^{\infty}$ imbedding then $\left(S^{n}, f\left(S^{n-1}\right)\right)$ is diffeomorphic to $\left(S^{n}, S^{n-1}\right)$ (where $S^{n-1}$ is considered as the equator of $S^{n}$ ). The $C^{\infty}$ Schönflies Conjecture is valid for $n \neq 4$, by the combined results of $[1 ; 6 ; 7$ and 9$]$; so Proposition 1 and Theorem $5^{\prime}$ are valid for $n \neq 4$.

Theorem 2 is an immediate consequence of Theorem $5^{\prime}$ and the Riemann Mapping Theorem because the Riemann Mapping Theorem provides a diffeomorphism between $R^{2}-S$ and $R^{2}-0$ since $\hat{R}^{2}-S$ and $\hat{R}^{2}-0$ are simply connected regions on $S^{2}=\hat{R}^{2}=$ the one point compactification of $R^{2}$.

5. Definition. Two subsets $A$ and $B$ of $R^{n}$ are $C^{1}$ equivalent if there exist neighborhoods $U$ and $V$ of $A$ and $B$ and a bi- $C^{1}$ homeomorphism of $U$ onto $V$ which takes $A$ onto $B$.

It is simple to see that if $A$ and $B$ are $C^{1}$ equivalent then both or neither are funnel-sections.

Definition. A continuum $A$ contained in $R^{n}$ is a small funnelsection if there exists $f \in F^{n}$ and $p=\left(t_{0}, y_{0}\right) \in R^{n+1}$ such that $K_{s}(p)$ is $C^{1}$ equivalent to $A$ for all $s \neq t_{0}$.

At first it might seem that very few continua are small funnelsections. For instance, for $n=2$ it might seem that the circle $S^{1}$ (or any nonsimply connected continuum in $R^{2}$ ) could not be a small funnel-section (cf. [8, pp. 238-239]). However, this is not the case. We have

THEOREM 6. $S^{1}$ is a small funnel-section.

6. The open questions about funnel-sections include:

1. What is a necessary and sufficient condition that a continuum be a funnel-section? 
2. Are all ANR's funnel-sections? (Relevant to this question are the facts that Theorem 2 shows that there exist funnel-sections which are not ANR's and that the $P$ of Theorem 1 is not an ANR.)

3 . Is the property of being a funnel-section a topological property? (It is easy to see that any continuum homeomorphic to $P$ is not a funnel-section.)

4. If the property of being a funnel-section is topological, then is it actually just a property of homotopy type? In particular, can some continuum of the same homotopy type as $P$ be a funnel-section?

5. Does there exist a continuum $A$ in $R^{m}$ which is an $n$-funnelsection for $n>m$ (i.e., $f \in F^{n}$ exists such that for some $p \in R^{n+1}$ and $s \in R, K_{s}(p)=i(A)$, but no such $f$ exists in $\left.\mathfrak{F}^{m}\right)$ ?

6. Does there exist a funnel-section which is not small?

7. What are some examples other than $P$ of continua which are not funnel-sections? Is there any reason that a continuum fails to be a funnel-section other than that it "incorporates the global spiral shape of $P$ ?" Is this at least true for continua in $R^{2}$ ?

\section{REFERENCES}

1. M. Brown, A proof of the generalized Schönflies conjecture, Bull. Amer. Math. Soc. 66 (1960), 74-76.

2. M. Fukuhara, Sur l'ensemble des courbes intégrales d'un système d'équations différentielles ordinaires, Proc. Imp. Acad. Japan, 6 (1930), 360-362.

3. E. Kamke, Zur Theorie der Systeme gewöhnlicher Differential-gleichungen. II, Acta Math. 58 (1932), 57-73.

4. M. Kervaire and J. Milnor, Homotopy spheres. I, Ann. of Math. (2) 77 (1963), 504-536.

5. H. Kneser, Ueber die Lösung eines System gewöhnlicher Differentialgleichungen das der Lipschitzchen Bedingung nicht genügt, S.-B. Preuss. Akad. Wiss. Phys. Math. Kl. (1923), 171-174.

6. B. Mazur, On imbeddings of spheres, Bull. Amer. Math. Soc. 65 (1959), 59-65.

7. J. Munkres, Obstructions to the smoothings of piecewise-differentiable homeomorphisms, Ann. of Math. (2) 72 (1960), 521-554.

8. M. Nagumo and M. Fukuhara, Un théorème relatif à l'ensemble des courbes intégrales d'un système d'équations différentielles ordinaires, Proc. Phys. Math. Soc. Japan (III) 12 (1930), 233-239.

9. S. Smale, On the structure of manifolds, Amer. J. Math. 84 (1962), 387-399.

JoHNS HopkINS UnIVERSITY 